\title{
AC-DC Continuous Mode Power Converter with Frequency Compensation for Low Cost and High Efficiency Led Lamps
}

\author{
Araújo C.M.B¹, Berci C.D², Guelfi A. E ${ }^{3}$, Boscoli M.E.A ${ }^{4}$, and Donzelli D. ${ }^{5}$
}

\begin{abstract}
This paper presents a new architecture of a high brightness LEDs driver, with low cost and high yield. Employs a CUK type converter in continuous mode with current compensation rate to feed the high-brightness LEDs through the grid, from $85 \mathrm{~V}$ to $265 \mathrm{~V}$, operating at high frequency switched Pulse Width Modulation (PWM) and connected to a series LC resonant filter for load feeding entry in protection against voltage surges. Twenty LEDs High Power OSRAM 720-GWPSL and a circuit with constant current in Offline mode were used. The proposed architecture aims to present a proposal for a low-cost and low current consumption driver. The comparative study between the prototype of this device from the graphs obtained from the results of laboratory measurements.
\end{abstract}

Keywords - CUK Converter, LED Lamp, Continuous Current Mode, Pulse Width Modulation (PWM).

\section{INTRODUCTION}

A S DEA 03/11 technical note from the Brazilian Energy Research Company, which has a projection of electricity demand for the next 10 years, in the medium-term 2011-2020 the consequences of the international financial crisis will be strongly in the behavior of Brazilian power sector with sharp fall in industrial electricity consumption with a sharp power pricing increase in the first months of 2015 for all consumers classes. In this scenario, it is very important the development of more efficient electrical equipment and materials, and with low-cost, for enabling the use of LED lighting technology on a large scale. LEDs (Light Emitting Diodes) are composed of two different semiconductor materials which form a PN junction allowing the current flow in only one direction. If the PN junction is forward biased, the electrons gaps start to move in the $\mathrm{P}$ layer and the $\mathrm{N}$ layer toward the depletion region (transition area between the $\mathrm{P}$ and $\mathrm{N}$ materials). Near that region, the recombination of electrons and holes generate energy that is released in the form of light photons [1].

All the authors are with Department of Electrical Engineering, Electroeletronic and Instrumentation Laboratory (EIL), University of Western São Paulo (UNOESTE), Campus II - Presidente Prudente, 19067-175 Sao Paulo (Brazil).
In Fig. 1 is shown the simplified physical structure of the LED and the light emission process [2].

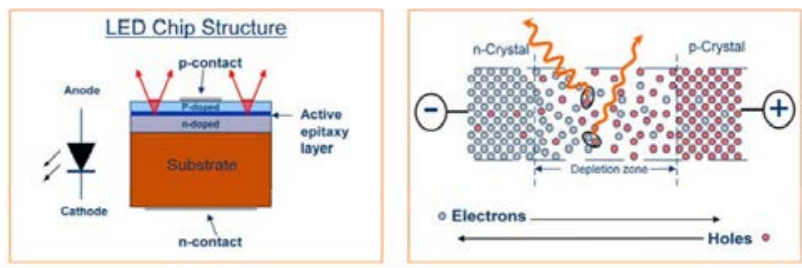

Fig. 1 Physical structure and behavior representation of a LED component [2]

Fig. 2 shows the constructive representation of the LED's in the prototype. Inside the cavity LED it is a semiconductor chip mounted on a frame of conductive terminals that are housed in a pre-molded encapsulation. The terminal conductors act as a path for thermal dissipation for the heat from the semiconductor switch, and also serves as electrical and mechanical interface to the Printed Circuit Board (PCB). A gold wire is used for bonding the upper side of the tablet to the anode (A) and cathode (C) side terminal leads. The housing cavity is filled with epoxy resin and serves as a reflector for the extraction of the maximum amount of light to the outside of the package.

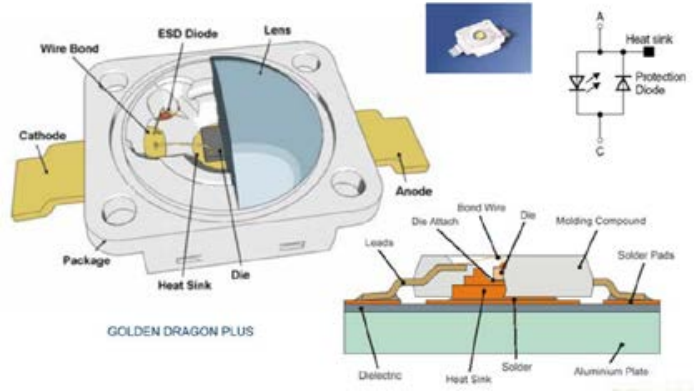

Fig. 2 Hi-power LED GW PSLRS1.EC [2]

In the prototype presented in this article was used nine LEDs model GW PSLRS1.EC from [2]. The LEDs are powered by a four stages driver. The first stage consists of a resonant filter RLC (Resistor-Inductor-Capacitor) and a voltage surge protection (varistor and fusistor), the second stage is a rectifier AC-DC, the third stage has a primary switch with $12 \mathrm{~W}$ power and switching frequency fixed at $42 \mathrm{kHz}$. The last stage consists of an output filter to reduce current ripple levels to an acceptable value [3] as the standard [4] and [5]. 


\section{METHODS}

The proposed LED lamp aims to be a reliable substitute for now used incandescent or even compact fluorescent lamps.

Such lamps have power from 9W up to $100 \mathrm{~W}$ and present luminosity efficiency about 55 to $70 \mathrm{~lm} /$ watt. The subject of this work is to create a lamp with medium power and high efficiency, than was defined the use of 9 LED's units, and expected luminous flux up to $1100 \mathrm{~lm}$.

The lamp power is less than $11 \mathrm{~W}$ given a luminosity efficiency greater than $100 \mathrm{~lm} / \mathrm{W}$. Such efficiency is higher than common led lamps that can be easily found now days.

Its high efficiency is due, among others, to the superior efficiency of the LED's itself and also to the controller design.

The Table I shows the main data about the LED used in this project:

TABLE I

MAIN FEATURES OF LED (GW PSLRS1.EC-LPLR-5U8X-1)

\begin{tabular}{|c|c|c|c|}
\hline Parameter & Symbol & Value & Unit \\
\hline Operation temperature limit & $\mathrm{T}_{\mathrm{oe}}$ & -40 a 110 & ${ }^{\circ} \mathrm{C}$ \\
\hline Junction temperature & $\mathrm{T}$ & 125 & ${ }^{\circ} \mathrm{C}$ \\
\hline Forward Current & $\mathrm{I}_{d}$ & 10 a 200 & $\mathrm{~mA}$ \\
\hline Reverse Current & $\mathrm{I}_{R}$ & 20 & $\mathrm{~mA}$ \\
\hline Surge Current & I $S$ & 300 & $\mathrm{~mA}$ \\
\hline $50 \%$ of Vision Angle & $2 \phi$ & 120 & $\circ$ \\
\hline $\begin{array}{ll}\text { Voltage } & {[\min ]} \\
& {[\text { tip }]} \\
& {[\max ]}\end{array}$ & $\mathrm{V}_{d}$ & $\begin{array}{l}5.60 \\
6.10 \\
6.40\end{array}$ & $\begin{array}{l}\mathrm{V} \\
\mathrm{V} \\
\mathrm{V}\end{array}$ \\
\hline Color temperature & $\mathrm{K}$ & 2700 & - \\
\hline $\begin{array}{l}\text { Luminous Flux } \\
I_{F}=150 \mathrm{~mA}\end{array}$ & $\Phi \mathrm{V}$ & $\begin{array}{l}112 \\
121\end{array}$ & $\operatorname{lm}$ \\
\hline Luminoues Intensity & $\mathrm{I}_{L}$ & 39,6 & $\mathrm{~cd}$ \\
\hline Equivalent Resistence & $\mathrm{R}_{E Q}$ & 1,8 & $\Omega$ \\
\hline
\end{tabular}

Using the data from LED's manufactory were obtained a total voltage drop (V) of $54,9 \mathrm{~V}$ and an equivalent resistance (Rs) of $16.2 \Omega$ for the set of nine LEDs. In this case, it is recommended to use a transformer with the secondary voltage of $60 \mathrm{Vrms}$. In equation 1 shows the electrical model for the LED used:

$V_{L E D}=\frac{\eta \cdot k \cdot T}{q} \cdot 1 n\left(\frac{{ }^{I} L E D}{I_{S}}\right)+R s \cdot{ }^{I} L E D$

Equation 1

Where:

$V_{L E D}=$ LED forward voltage, Volts (V);

$\eta$ = Ideality factor;

$k$ = Boltzmann constant, $1.3805 .10^{-23}(i / k)$;

$\mathrm{T}=$ Temperature, Kelvin $(k)$;

q= Eletron charge, Eletron-Volt, $1.602 .10^{-19}$ Coulomb (C);
${ }^{I} L E D=$ LED forward current, Amperes (A);

$I_{S}=$ Saturation forward current, Amperes (A).

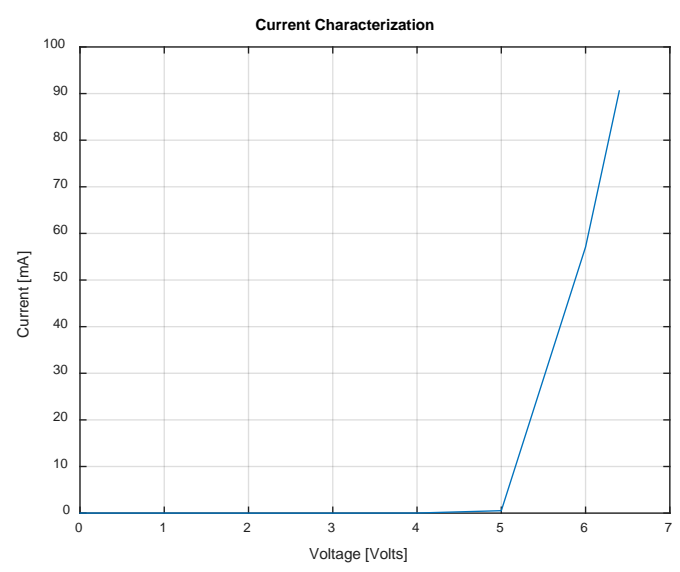

Fig. 3 Current Characterization for a GW PSLRS1.EC LED

The Fig. 3 shows the current characterization test for a unit of the GW PSLRS1.EC LED [2] using a Source and Measure Unit (SMU) Model U2723 - Agilent Technologies, with provide a current test up to $120 \mathrm{~mA}$. In the results obtained was used a limit current of $100 \mathrm{~mA}$ to avoid power saturation.

Even was not possible to get the full scale response using the available equipment, the results found shows the correct behavior of the tested units regardless the data sheet.

Searching for theoretical foundations was made a bibliographical research, mainly about AC-DC converters, subject of this paper. A theoretical study about AC-DC convert with "CUK" topology and operating in continuous current mode was developed. The main idea is to emphasize the prototype efficiency in face of bulb type LED lamps used now days, in addition to describing the design and dimensioning of the constituent elements of it. To elucidate the design steps, some studies cases was made and the results disposed in tables to display data from these studies in order to compare their data.

\section{THE Non IsOlated CUK CONVERTER}

The switch converter developed for the LED lamp operates at $42 \mathrm{kHz}$ frequency, which makes reduced its volume.

The converter is used to produce the DC voltages with better characteristics regarding noise emitted by the converter in both sides, input voltage $\left(\mathrm{V}_{\mathrm{e}}\right)$ and the output voltage $\left(\mathrm{V}_{\mathrm{s}}\right)$. The output has low noise due to non pulsating characteristic of the current in the inductor [3].

To the converter are used two inductors, one at the input and another at the output, providing a low ripple in both sides, due the inductor inertial current features.

The Fig. 4 illustrates the electrical diagram of the proposed circuit, wish conception follows the Ridley model [7]. 


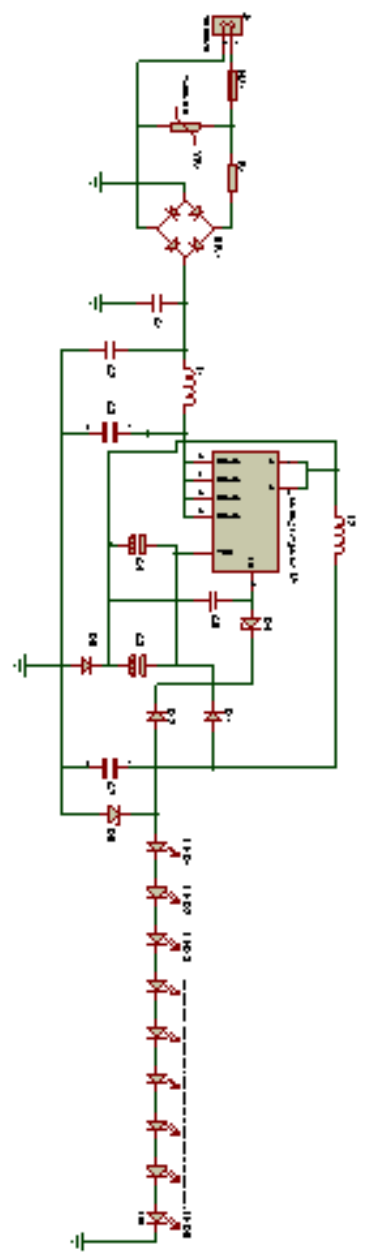

Fig. 4 Electrical diagram of the proposed CUK converter

In [7], presented a sampling model for the current-mode control, modeling the converter power stage using a third order polynomial form resulting in a low frequency pole $\omega_{p}$ and a double pole $\omega_{n}$ at a full cycle switching frequency.

For a more precisely analysis, the converter model was numerically implemented using MATLAB $^{\circledR}$ software. The results of this study where shown as follow:

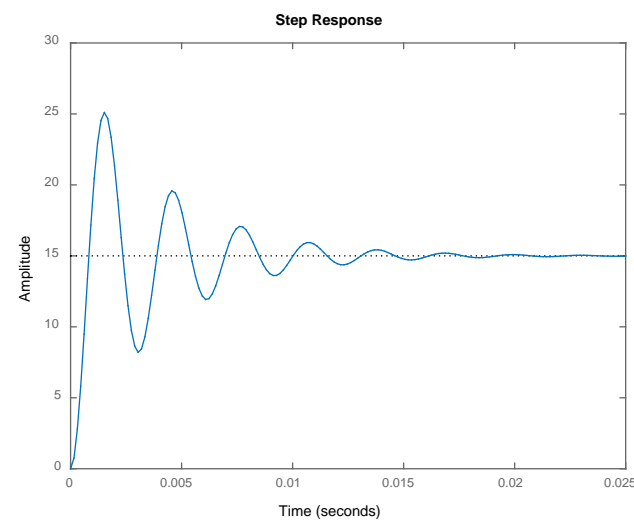

Fig. 5 Step response of the numerical model

The step response (Fig. 5) allow us to identify some o main features of the proposed controller:

$$
\begin{array}{lll}
\checkmark & \mathrm{T}_{\mathrm{r}}=8,25 \cdot 10^{-4}(\mathrm{~s}) & \text { Rising time; } \\
\checkmark & \mathrm{T}_{\mathrm{s}}=0,0110(\mathrm{~s}) & \text { Settling time; } \\
\checkmark & \mathrm{T}_{\mathrm{p}}=0,0015(\mathrm{~s}) & \text { Peak time; } \\
\checkmark & \mathrm{T}_{\mathrm{d}}=5,26 \cdot 10^{-4}(\mathrm{~s}) & \text { Delay time; } \\
\checkmark & M_{p}=67,34 \% & \text { Max overshoot. }
\end{array}
$$

The frequency behavior of the controller is illustrated in the Fig. 6:

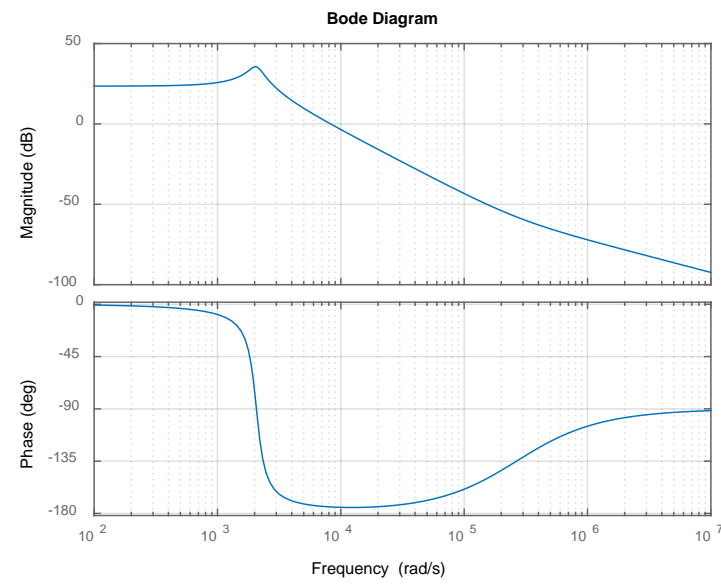

Fig. 6 Bode diagram

Is clear that the controller has a main pole at 2000rad/s (about $320 \mathrm{~Hz}$ ) and for low frequencies (less than 1000rad/s) the output gain is constant.

The proposed controller stability feature is shown in Fig. 7, where one can easy observe that there is no closed loops around the point -1 , which means that the control is stable for every gain $H(s)$.

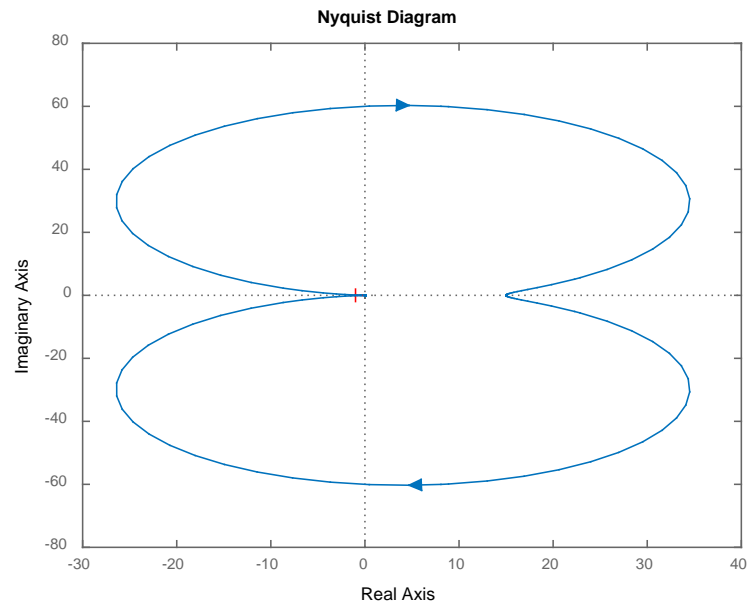

Fig. 7 Nyquist Diagram

The Fig. 8 presents the medium frequency measured in lab for the switching inductor for a constant output current. In such case, this inductor acts as a second order equivalent low filter. 


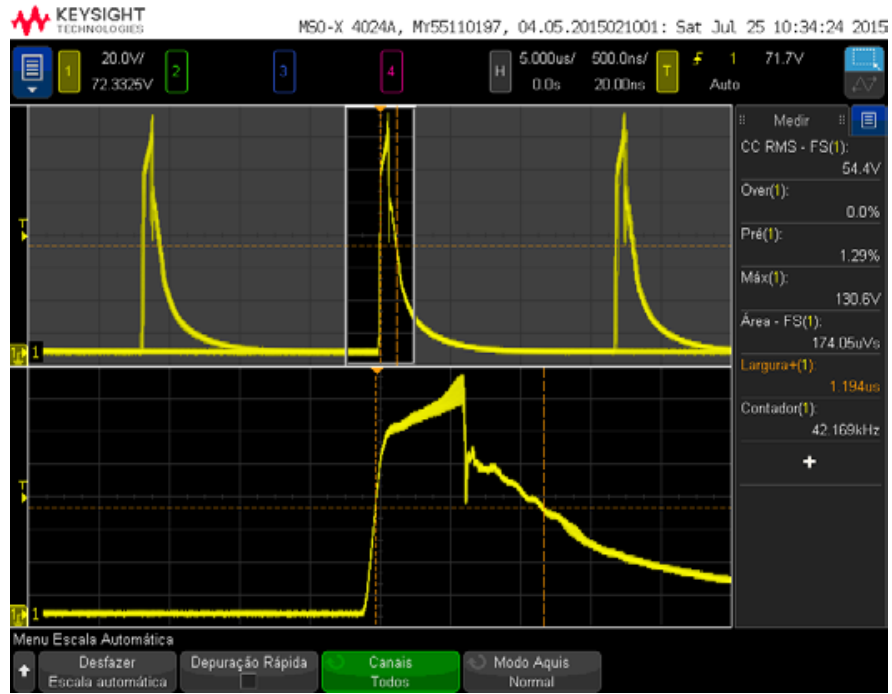

Fig. 8 Inductor frequency

\section{RESUlts}

The proposed CA-CC converter and the lamp itself were implemented in the Electroeletronic and Instrumentation Laboratory (EIL). The Table II shows the main electronic components used in the prototype assembly.

TABLE II

MAIN ELECTRONICS COMPONENTS UsEd IN PROTOTYPE

\begin{tabular}{ll}
\hline \hline \multicolumn{1}{c}{ Parameter } & \multicolumn{1}{c}{ Description } \\
\hline Pulse width modulation (PWM) & VIPEr22AS IC \\
Input Inductor & $1 \mu \mathrm{H}-100 \mathrm{~mA}$ \\
Output Inductor & $1 \mu \mathrm{H}-200 \mathrm{~mA}$ \\
Input Surge Protection & EPCOS $250 \mathrm{~V}$ Varistor \\
Full Cycle Rectifier & DF06 Rectifier bridge \\
Input Protection & $1 \mathrm{~A}-250 \mathrm{~V}$ fuse \\
Output Protection & $1.3 \Omega(\mathrm{Ohm})$ Resistor \\
\hline
\end{tabular}

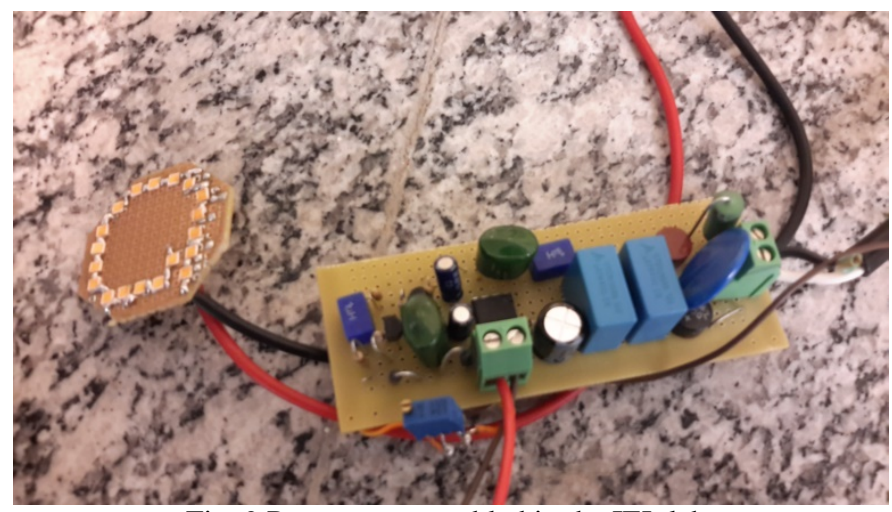

Fig. 9 Prototype assembled in the IEL lab

As can be seen in Fig. 9 the prototype was built on standard printed circuit board (matrix), with few components and with a low implementation cost. A very important advantage of the developed circuit is that it has not a transformer, due the voltage limiting performed by a capacitor and the regulation by an integrated circuit [8], the avoidance of a transformer became possible, reducing the circuit weight and dimensions, and even the material costs. Fig. 10 shows the average illuminance test performed with the prototype, where the photometric performance of the LEDs array was demonstrated by measuring the light using a digital Luxmeter. Measurements were made with light source from $100 \mathrm{~cm}$ of the Digital Luxmeter and sensor located in 6 different positions. It was obtained an average illuminance of $843 \mathrm{~lx}$ (lux). In Fig. 11 we have the lighting of the LEDs set, where there one can easily notice to uniform brightness of the entire set.

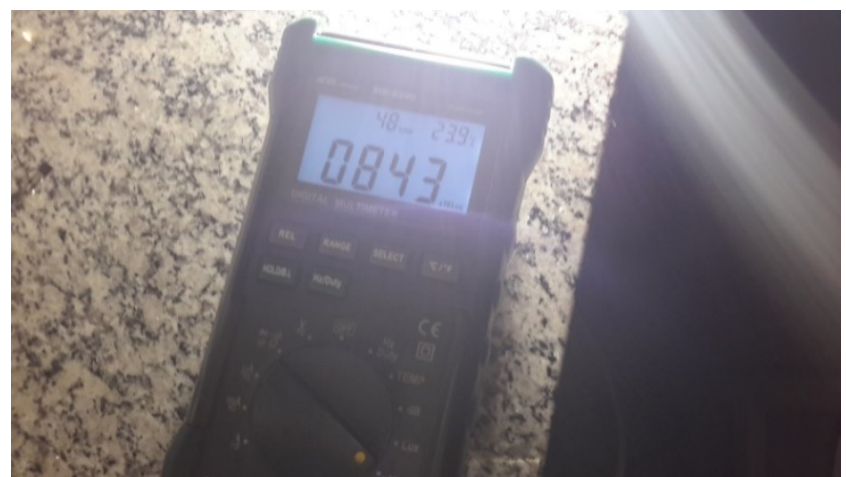

Fig. 10 Average illuminance (lux)

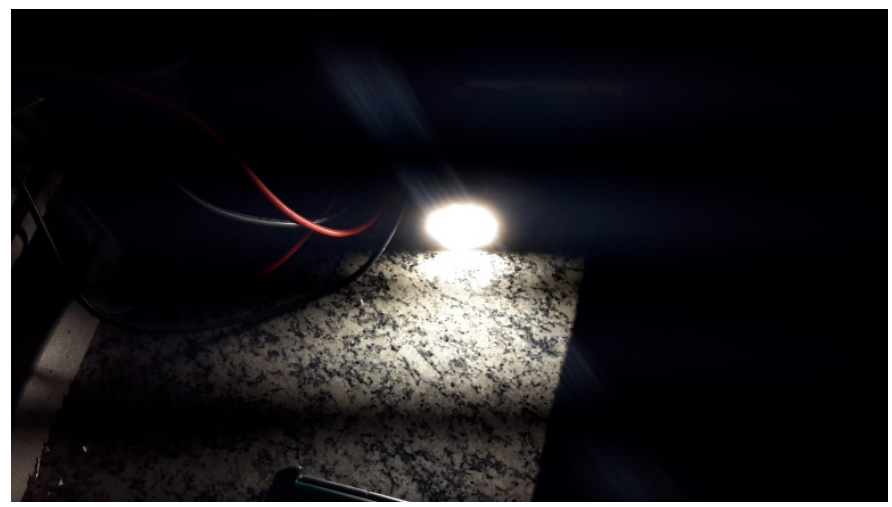

Fig. 11 LED's and its illumination during the tests

\section{CONCLUSIÓN}

In this paper we present the design of an low cost and high illuminance LED lamp with driver. All project development stages and experimental results have been shown using the developed prototype. Tests were also performed to evaluate the electrical and photometric efficiency.

In tests was proven the high efficiency aimed by the prototype, both in power consumption up to $189 \mathrm{~mA}$ load current, and also in the photometric efficiency 843lx recorded as average value of the measurements.

As future work the IEE research group will produce a bulbtype lamp, using a 3D printer and printed circuit board with surface mounted electronic components to archive a smaller circuit and a commercial design.

\section{REFERENCES}

[1] Bullough, J. D. Lighting Answers: Led Systems. National Lighting Product Information Program, Lighting Research Center, Rensselaer Polytechnic Institute. Vol. 7, Issue 3, 2003.

[2] OSRAM Opto Semiconductors. Datasheet DURIS S 5 - Version 1.0. Regensburg: OSRAM, 2014. 
[3] RASHID, Muhammad H. Power Electronics: Circuits, Devices \& Applications (4th Edition). Makron, 1999.

[4] SAKTHIVEL, K. N.; DAS, Sisir K.; KINI, K. R. Importance of quality AC power distribution and understanding of EMC standards IEC 61000-3-2, IEC 61000-3-3 and IEC 61000-3-11. In: Electromagnetic Interference and Compatibility, 2003. INCEMIC 2003. 8th International Conference on. IEEE, 2003. p. 423430.

[5] BILLINGS, Keith H. Switchmode power supply handbook. McGrawHill Professional, 1999.

[6] KASSAKIAN, John G.; SCHLECHT, Martin F.; VERGHESE, George C. Principles of power electronics. Reading, USA: Addison-Wesley, 1991.

[7] R.B. Ridley. A new continuous-time model for current-mode control. IEEE Trans. Power Electron., 6 (2) (1991), pp. 271-280. http://dx.doi.org/10.1109/63.76813

[8] STMicroelectronics. Datasheet VIPEr22AS. Italy: STMicroelectronics, 2002. 